\title{
Increased QT Dispersion and High Risk of Ventricular Arrhythmias is Associated with Hyperuricemia in Individuals with Normal Renal Function
}

\author{
Bülent Huddam ${ }^{1}$, Alper Alp ${ }^{1}$, Dilek Gibyeli Genek ${ }^{1}$, Alper Azak², Volkan Karakus ${ }^{3}$ \\ ${ }^{1}$ Department of Nephrology, Faculty of Medicine, Mugla Sıtkı Koçman University, Mugla, Turkey \\ 2 Department of Nephrology, Atatürk City Hospital, Ministry of Health, Balikesir, Turkey \\ 3 Department of Hematology, Faculty of Medicine, Alanya Alaaddin Keykubat University, Alanya/Antalya, Turkey
}

\section{ABSTRACT}

Background and aim: Uric acid elevation has been shown to be an important risk factor for cardiovascular and cerebrobascular disease. QT dispersion (QTd) is a parameter that shows the heterogeneity of ventricular repolarization and can be calculated noninvasively from surface electrocardiography. Increased QTd has been associated with severe arrhythmia and risk of sudden death in many patients and disease groups. In this context, we aimed to investigate the effect of uric acid levels on QTd and the effects of decrease in uric acid levels on QTd. Methods: A total of 225 patients with normal renal function were included in the study; 133 of these patients were hyperuricemic (>7 mg/dL), and the remaining 72 patients were normouricemic (Group 1). The hyperuricemic patients were randomly divided into 2 groups, one group $(n=67)$ was given placebo (Group 2) for 4 months, and the remaining 66 patients were given allopurinol $300 \mathrm{mg} /$ day (Group 3). Results: Hyperuricemic patients had higher hsCRP and QTd and lower eGFR values compared to the normouricemic control group. After 4 months of treatment, 66 patients treated with allopurinol showed a significant decrease in serum uric acid, systolic and diastolic blood pressure, and hsCRP levels, and a significant increase in eGFR. Although the QTd values in the treatment group did not decrease to the same levels as in the normouricemic control group, a statistically significant decrease was found compared to their baseline values. In hyperuricemic control and normouricemic control patients, there were no differences in the levels of uric acid, hsCRP, eGFR, systolic and diastolic blood pressure, and QTd values compared to baseline values. Conclusions: There was a significant association between elevated serum uric acid and QTd, as well as with inflammatory biomarkers. Also, patients who had received hypouricemic therapy during the follow-up period presented a significant decrease in inflammatory markers as well as QTd. This indicates the beneficial effects of decreasing uric acid levels in decreasing the risk for future major adverse events related to ventricular arrhythmias.

Keywords: electrocardiography, allopurinol, uric acid, acute ventricular arryhthmias

\section{ARTICLE HISTORY}

Received: March 16, 2021

Accepted: May 23, 2021

\section{CORRESPONDENCE}

\section{Alper Alp}

Department of Nephrology, School of Medicine, Mugla Sitki Kocman University

Kötekli Mahallesi, Marmaris Yolu üzeri, No: 48, 48000 Menteşe/Muğla, Turkey Tel: +90 $5303102244 /+902522141323$ E-mail: alperalp@mu.edu.tr 


\section{INTRODUCTION}

In electrocardiographic (ECG) examination, the time from the start of the $\mathrm{Q}$ wave to the return of the $\mathrm{T}$ wave to the isoelectric line is called the QT distance, which ranges from 0.35 to 0.44 seconds in adults. The difference between the longest QT distance and the shortest QT distance is called the QT dispersion (QTd), which are corrected according to the paitent's heart rate. QTd is a parameter that shows the heterogeneity of ventricular repolarization and can be calculated noninvasively from surface electrocardiography.

Ventricular fibrillation, ventricular tachycardia (VT), and Torsades de Pointes are also referred to as malignant ventricular arrhythmias and are the leading factors of sudden cardiac death. Several quantifiable ECG entities may predict such acute and severe clinical situations including a long QTc, QTd, Tpeak-Tend (Tp-e), Tp-e dispersion, and Tp-e/QT. Increased QTd has been associated with an increased risk for developing severe ventricular arrhythmias and sudden cardiac death in many patients and disease groups. ${ }^{1-3}$ Therefore, analyzing these parameters is very important for the identification of patients at high risk of mortality.

Uric acid is the final product of the metabolism of purines, which is released as a result of the catabolism of nuclear material. ${ }^{4}$ Uric acid is an effective extracellular radical scavenger and also stimulates granular adhesion to the endothelium and the release of peroxide and superoxide free radicals. ${ }^{4,5}$ Uric acid elevation has been shown to have a high risk potential for cardiovascular diseases such as coronary heart disease, hypertension, peripheral vascular disease, heart failure, metabolic syndrome, and stroke. ${ }^{6-9}$ In addition, a close relationship between high serum uric acid levels and circulating inflammatory markers has been observed..$^{10,11}$ The role of uric acid in the development of cardiovascular disease has not been fully explained. However, uric acid is known to cause frank ECG changes such as ischemic alterations, left ventricular hypertrophy (LVH), sinus tachycardia, and ventricular tachyarrhytmias, especially in LVH patients.

In this view, the present study aimed to examine the effects of uric acid levels on QTd and also the effects of reduction in uric acid levels on QTd.

\section{METHODS}

We counducted a prospective randomized study in Ankara Training and Research Hospital. The study protocol was approved by the local ethics committee and conducted in accordance with the ethical principles set by the Helsinki Declaration. All participants were included in the study after signing informed consent forms. A total of 225 patients with normal kidney function were included in the study, and 205 completed the study. The study population was divided into 3 groups as follows: Group 1 - normouricemic subjects $(n=72)$; Group 2 - hyperuricemic patients that had received placebo for 4 months, for treating the increased levels of uric acid ( $n=67)$; Group 3 - hyperuricemic patients that were administered $300 \mathrm{mg}$ of allopurinol daily for 4 months $(n=66)$. Patients with concomitant infection, chronic disease other than hypertension, known coronary artery disease (those with myocardial infarction, history of coronary artery bypass graft and percutaneous transluminal coronary angioplasty), valve disease, arrhythmia, branch block, kidney damage, electrolyte imbalance, and thyroid function disorders, patients who were on antihypertensive drugs or those who used drugs known to affect heart rate and ECG findings, as well as those who used alcohol or cigarettes were excluded from the study.

At the beginning of the study and at the end of the 4 th month, after 12 hours of fasting, blood glucose, urea, creatinine, ALT, AST, total cholesterol, triglyceride, highdensity lipoprotein (HDL), low-density lipoprotein (LDL), sodium, potassium, chloride, calcium, magnesium, hemogram, and C-reactive protein were measured in all patients. The estimated glomerular filtration rate (eGFR) was calculated using the Cockcroft-Gault formula. According to the results, those with abnormal findings in liver enzymes, altered blood cell count and plasma potassium, calcium, and magnesium levels were excluded.

Twelve-lead surface electrocardiograms of all patients were recorded and heart rate, RR, and QT distances were measured. The QT interval was considered as the distance from the beginning of the QRS complex to the end of the $\mathrm{T}$ wave. QT (QTc) values corrected for heart rate from QT distances were calculated with the Hodger formula (QTC $=[\mathrm{QT}+1.75 \times($ heart rate -60$)])$. QTd was calculated by taking the difference between the shortest average QT distance in all leads and the longest average QT distance. Corrected QT dispersion (QTcd) values were calculated in the same way.

\section{STATISTICAL ANALYSIS}

SPSS (Statistical Package for Social Sciences) for Windows, version 15.0 (SPSS Inc., Chicago, USA) was used for statistical analysis. Descriptive statistical methods (mean, standard deviation) and Student's $t$ test were used to com- 
TABLE 1. Baseline characteristics and laboratory findings across the three study groups

\begin{tabular}{lcccc}
\hline & $\begin{array}{c}\text { Normouricemic } \\
\text { group } \\
\mathbf{( n = 7 2 )}\end{array}$ & $\begin{array}{c}\text { Hyperuricemic- } \\
\text { placebo group } \\
\mathbf{( n = 6 7 )}\end{array}$ & $\begin{array}{c}\text { Hyperuricemic- } \\
\text { allopurinol group } \\
(\mathbf{n}=\mathbf{6 6})\end{array}$ & p value \\
\hline Age (years, mean) & $48.4 \pm 9.2$ & $50.4 \pm 11.2$ & $54.4 \pm 8.0$ & 0.13 \\
Gender, n (\%) & $33(46)$ & $32(48)$ & $35(53)$ & 0.23 \\
Hemoglobin (g/dL) & $14.0 \pm 1.4$ & $13.9 \pm 1.6$ & $14.8 \pm 1.2$ & 0.07 \\
Uric acid (mg/dL) & $4.5 \pm 0.9$ & $8 \pm 1$ & $8.3 \pm 1.1$ & $<0.001$ \\
BMI (kg/m²) & $28.4 \pm 3.3$ & $29.7 \pm 3.4$ & $28.4 \pm 2.9$ & 0.26 \\
24-hour average SBP (mmHg) & $119.4 \pm 11.2$ & $123.2 \pm 13.5$ & $127.6 \pm 10.4$ & 0.08 \\
24-hour average DBP (mmHg) & $77.3 \pm 6.1$ & $75.6 \pm 8.7$ & $75.1 \pm 7.8$ & 0.06 \\
eGFR (mL/min/1.73 m $\left.{ }^{2}\right)$ & $92.8 \pm 13.7$ & $84.3 \pm 16.7$ & $86.3 \pm 19.4$ & 0.04 \\
Spot urine protein/creatinine & $0.11 \pm 0.04$ & $0.12 \pm 0.13$ & $0.12 \pm 0.09$ & 0.52 \\
hsCRP (mg/L) & $3.3 \pm 2.5$ & $6.9 \pm 3.4$ & $7.4 \pm 5.8$ & 0.002 \\
LDL-cholesterol (mg/dL) & $125.7 \pm 38.3$ & $113.5 \pm 34.4$ & $123.0 \pm 30.7$ & 0.44 \\
Glucose (mg/dL) & $89.8 \pm 7.9$ & $90.3 \pm 6.4$ & $94.3 \pm 6.9$ & 0.06 \\
QTd & $35.01 \pm 6.07$ & $46.08 \pm 17.38$ & $47.4 \pm 16.91$ & $<0.001$ \\
QTdc & $40.11 \pm 6.99$ & $56.20 \pm 19.67$ & $57.09 \pm 17.12$ & $<0.001$ \\
\hline
\end{tabular}

pare quantitative data between groups that showed normal distribution, and the Mann-Whitney U test was used for group comparisons between non-normally distributed parameters. In the comparison of qualitative data, the Chi-squared test was used. Spearman's rho correlation test was used to examine the relationships between parameters. The results were evaluated in the $95 \%$ confidence interval, and the significance level was $\mathrm{p}<0.05$.

\section{RESULTS}

Initial demographic, clinical, and laboratory data of the three groups are shown in Table 1 . There were no significant differences between the groups in regards to age, gender, blood pressure, and LDL cholesterol values. Hyperuricemic patients had higher hsCRP, QTd and lower eGFR values compared to the normouricemic control group.

After 4 months of treatment, the 66 patients treated with allopurinol showed a significant decrease in serum uric acid (Figure 1), systolic and diastolic blood pressure, and hsCRP values, and a significant increase in eGFR. Although QTd values did not decrease during follow-up in the normouricemic group, a statistically significant decrease in the QTd was found in patients that had received allopurinol treatment for 4 months. In the hyperuricemicplacebo and normouricemic patients, there were no differences regarding the levels of uric acid, hsCRP, eGFR, systolic and diastolic blood pressure, and QTd values compared to the baseline values (Table 2).

\section{DISCUSSIONS}

The present study aimed to show whether there is a significant difference between QTd values in asymptomatic hyperuricemic patients without a history of concomitant disease compared to control groups of subjects with normal uric acid levels, and whether there is a change in QTd values in patients that undergo hypouricemic treatment with allopurinol.

Prolonged QTd, which can easily be quantified in clinical practice by analyzing the 12-lead ECG tracing, is associated with a high incidence of major arrhythmic events

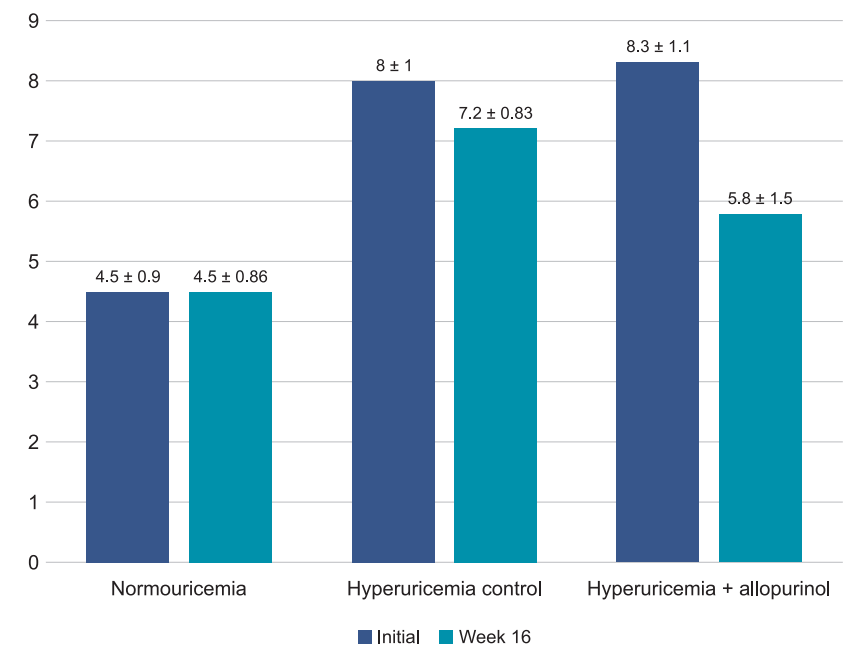

FIGURE 1. Mean serum uric acid levels ( $\mathrm{mg} / \mathrm{dL}$ ) across the three study groups at baseline and at the 16-week follow-up 
TABLE 2. Mean serum uric acid, QTd, mean systolic (SBP) and diastolic (DBP) blood pressure, measured at baseline and at the end of the 16-week follow-up. Analysis across the three study groups

\begin{tabular}{|c|c|c|c|c|c|c|c|c|c|}
\hline & \multicolumn{3}{|c|}{$\begin{array}{l}\text { Normouricemic group } \\
\qquad(n=72)\end{array}$} & \multicolumn{3}{|c|}{$\begin{array}{l}\text { Hyperuricemic- } \\
\text { placebo group } \\
(\mathrm{n}=67)\end{array}$} & \multicolumn{3}{|c|}{$\begin{array}{l}\text { Hyperuricemic-allopurinol group } \\
\qquad(\mathrm{n}=66)\end{array}$} \\
\hline & Initial & Week 16 & $p$ value & Initial & Week 16 & p value & Initial & Week 16 & p value \\
\hline Uric acid (mg/dL) & $4.5 \pm 0.9$ & $4.5 \pm 0.86$ & 0.26 & $8 \pm 1$ & $7.2 \pm 0.83$ & 0.70 & $8.3 \pm 1.1$ & $5.8 \pm 1.5$ & $<0.001$ \\
\hline QTd & $35.01 \pm 6.07$ & $34.4 \pm 6.91$ & 0.18 & $46.08 \pm 17.38$ & $47.31 \pm 17.61$ & & $47.4 \pm 16.91$ & $37.22 \pm 14.91$ & $<0.001$ \\
\hline QTdc & $40.11 \pm 6.99$ & $39.52 \pm 5.91$ & & $56.20 \pm 19.67$ & $58.11 \pm 15.88$ & & $57.09 \pm 17.12$ & $44.76 \pm 18.01$ & $<0.001$ \\
\hline $\mathrm{eGFR}\left(\mathrm{mL} / \mathrm{min} / 1.73 \mathrm{~m}^{2}\right)$ & $92.8 \pm 13.7$ & $93.3 \pm 9.2$ & 0.63 & $84.3 \pm 16.7$ & $84.4 \pm 16.3$ & 0.77 & $86.3 \pm 19.4$ & $89.6 \pm 12.6$ & 0.001 \\
\hline hsCRP (mg/dL) & $3.3 \pm 2.5$ & $3.4 \pm 2.2$ & 0.79 & $6.9 \pm 3.4$ & $5.9 \pm 3.8$ & 0.04 & $7.4 \pm 5.8$ & $4.6 \pm 3.7$ & 0.003 \\
\hline Average SBP (mmHg) & $119.4 \pm 11.2$ & $116.4 \pm 13.4$ & 0.46 & $123.2 \pm 13.5$ & $116.8 \pm 9.9$ & 0.26 & $127.6 \pm 14.4$ & $116.9 \pm 11.7$ & 0.005 \\
\hline Average DBP (mmHg) & $77.3 \pm 6.1$ & $76.2 \pm 11.8$ & 0.39 & $75.6 \pm 8.7$ & $73.9 \pm 13.9$ & 0.22 & $75.1 \pm 7.8$ & $74.9 \pm 12.4$ & 0.09 \\
\hline
\end{tabular}

due to regional heterogeneities of repolarization. Nonhomogeneous or irregular conduction velocities or regional ventricular repolarization in different parts of the ventricles may reveal a tendency for severe and acute ventricular arrhythmias through a re-entry pathway (ventricular premature beats, VT, and ventricular fibrillation) that may lead to sudden cardiac death. This effect has been described in both healthy individuals and those with underlying cardiac diseases. ${ }^{12-14}$ In a study with a long follow-up period, Cuddy et al. showed that in healthy men, increased heart rate and prolonged QTd, detected in ECG records 5 years before the event, were associated with sudden unexpected death. ${ }^{15}$ In a meta-analysis reported by Bazoukis et al., on 22 studies, it was shown that QTd was significantly associated with arrhythmic episodes and allcause mortality in patients with coronary artery disease and heart failure. Also, in this study, a significant correlation was found between higher QTd and the incidence of major arrhythmic episodes in patients with myocardial infarction. ${ }^{16}$ In the ELITE heart failure study, in patients with heart failure treated with losartan, a decrease in sudden cardiac death was shown in comparison with those treated with captopril. This result was shown to be caused by increased QTd in the group receiving captopril. ${ }^{17}$ In addition, patients with hypertrophic cardiomyopathy that present ventricular arrhythmias have been reported to have significantly higher QTd compared to those without ventricular arrhythmia. ${ }^{18}$

In recent years, besides the traditional risk factors of atherosclerosis, new risk factors have been emphasized. One of them is high serum uric acid level. The relationship between coronary heart diseases and hyperuricemia was first shown in a cross-sectional study conducted in 1951. ${ }^{19}$ Subsequent epidemiological studies revealed that an increase in uric acid levels is an important risk factor for mortality in cardiovascular diseases. However, there is still ambiguity in regards to the pathophysiological mechanism via which increased uric acid levels may influence patient outcomes. In addition to studies that identify uric acid as an independent marker of coronary heart disease, there are still conflicting evidence in this regard. ${ }^{20-28}$

It is known that uric acid has an antioxidant-like effect in the early stages of the atherosclerotic process and is one of the strongest contributing factors of plasma antioxidant capability. However, this antioxidant-like effect becomes paradoxically pro-oxidant at high serum uric acid levels in the late phases of atherosclerosis. This paradoxical condition seems to depend on many environmental aspects such as the stage of the disease, tissue and substrate localization, acidity, reduction of other local antioxidants, and the state of oxidant and enzyme release/availability. ${ }^{29}$ In this context, the importance of xanthine oxidase (xanthine $\rightarrow$ uric acid) activity level is also emphasized. Xanthine oxidase increases oxidative stress by stimulating uric acid production, and, independent of this effect, it is considered that upregulation of this pathway may cause vasoconstriction and a decrease in myocardial function. ${ }^{30}$ Thus, allopurinol, which inhibits xanthine oxidase, prevents both uric acid production and oxidative stress. These bilateral positive effects have been shown to reduce cardiovascular complications after coronary artery bypass operation and in subjects with dilated cardiomyopathy. ${ }^{31,32}$ Allopurinol has been shown to inhibit the reduction of nitric oxide production in patients with hypertension, diabetes, and heart failure. ${ }^{32-34}$ Doehner et al. showed that the uric acid reduction potential of allopurinol was very strongly associated with the improvement of endothelial function. ${ }^{33}$ In animal studies by Mazzali et al., uric acid has been shown to be an important factor in decreasing nitric oxide production, thus causing vascular changes in 
the kidney and hypertension. ${ }^{35,36}$ Nitric oxide production has also been shown to decrease in mouse models with short-term hyperuricemia (one week before the onset of hypertension)..$^{36}$ All these studies indicated the potentially harmful effects of uric acid on endothelial function and properties. In contrast to those described above, Waring et al. reported that uric acid did not have a direct role in endothelial dysfunction. In their study, healthy men were injected with uric acid solution, which did not lead to a disruption in cardiovascular function and was no associated with acetylcholine-induced vasodilation and alteration of endothelial nitric oxide release. ${ }^{37}$ However, a significant limitation of this study was that patients with an existing oxidative stress, such as heart failure or vascular disease, were not included. Cardiovascular diseases are mostly associated with endothelial dysfunction, the formation of free oxygen radicals, and proinflammatory conditions. ${ }^{38}$ What is the main role of uric acid in this relationship? Is it protective, ineffective, or harmful? This issue has not been adequately addressed so far. Oxidants produced by the formation of xanthine oxidase and uric acid may have negative effects on the vascular system, and there is significant evidence that uric acid itself may have undesirable effects that are not directly related to oxidant formation. ${ }^{34,36}$ In a large population study on healthy individuals evaluating the relationship between uric acid levels and abnormal ECG findings, high uric acid levels were correlated with findings of previous myocardial infarction, left ventricular hypertrophy, sinus tachycardia, and tachyarrhytmias. ${ }^{39}$ In addition, Özyllmaz et al. reported that high uric acid level was associated with an increase in the frequency of VT in patients with hypertrophic cardiomyopathy. $4^{0}$

The exact mechanisms that increase the risk of QTC prolongation are still unclear. However, it is plausible that high uric acid levels might adversely affect depolarization and repolarization by causing subclinical endomyocardial changes. It has been stated that uric acid causes cardiac damage by multiple pathophysiological mechanisms: (1) direct endothelial damage; (2) abnormalities in the coronary microvascular circulation (reduction in endotheliumdependent vasodilation with adverse effects on NO); (3) intracellular electrolyte disturbances, which increase the risk of potential arrhythmias; (4) increase in microvascular inflammation and its end-products; (5) activation of the renin-angiotensin system, which leads to proliferation in vascular smooth muscle cells, cardiac hypertrophy, and myocardial interstitial fibrosis. Increased intracellular levels of uric acid correlate with increased activation of some inflammatory markers (such as nuclear factor $\mathrm{kB}$, Ang II, IL-1 beta) and chemokines such as mitogen-acti- vated protein kinases. In experimental models, TNF-alpha and IL-1 beta have been shown to increase the potential of arrhythmia in ventricular myocytes by increasing $\mathrm{Ca}^{2+}$ escape. In a study evaluating the effect of serum uric acid level on ventricular arrhythmia, Nodera et al. evaluated VT attacks in patients with severe left ventricular heart failure (mean LVEF $31.2 \pm 10.1 \%$ ) who received ICD therapy. The results revealed that patients with higher serum uric acid levels ( $<6.1 \mathrm{mg} / \mathrm{dL}, \mathrm{n}=29 \mathrm{vs}$. $\geq 6.1 \mathrm{mg} / \mathrm{dL}, \mathrm{n}=27$ ) showed more frequent VT attacks..$^{41}$ In a study conducted by Yamada et al. among patients with left ventricular hypertrophy, which is considered an important risk factor for ventricular tachyarrhythmia and sudden cardiac death, they found more frequent VT attacks in patients with high uric acid levels. ${ }^{42}$ Pragmatically, oxidative stress may cause VT (which requires remodeling in ventricular structure and conductivity for its development) due to its effects on focal activity and reentry. So it seems reasonable to say that se $\neg$ rum uric acid, as a marker of oxidative stress and inflammation, may be associated with this serious entity.

In our study, the relationship between serum uric acid elevation and QTd, and the improvement of this relationship with allopurinol treatment revealed a different dimension of the link between uric acid and cardiac diseases. As a result of this study, it would not be speculative to say that QTd prolongation is highly likely in asymptomatic hyperuricemic patients and this may cause cardiacrelated mortality and morbidity.

\section{CONCLUSIONS}

Increased uric acid levels were associated with an increase in the QT interval dispersion, which is associated with an increased risk of malignant ventricular arrhythmia and sudden cardiac death. Also, patients with elevated serum uric acid that had received hypouricemic treatment presented a significant decrease in inflammatory biomarkers and in QTd, which indicates a positive impact on patient outcomes. Although there is still no global consensus in this regard, high serum uric acid levels may play an important role in disease progression and in the risk of potentially fatal arrhythmia. Controlled studies on larger populations with different cardiovascular profiles are required in order to validate the effect of hypouricemic agents in decreasing the risk of major adverse events related to ventricular arrhythmias.

\section{CONFLICT OF INTEREST}

None declared. 


\section{ACKNOWLEDGEMENT}

We are grateful to Ali Eroglu for his technical support.

\section{REFERENCES}

1. Somberg JS, Molnar J. Usefulness of QT dispersion as an electrocardiographically derived index. Am J Cardiol. 2002;89:291-294. doi: 10.1016/s0002-9149(01)02230-5.

2. Yu Z, Chen Z, Wu Y, et al. Electrocardiographic parameters effectively predict ventricular tachycardia/fibrillation in acute phase and abnormal cardiac function in chronic phase of ST-segment elevation myocardial infarction. J Cardiovasc Electrophysiol. 2018;29:756-766. doi: 10.1111/jce.13453.

3. Malik M, Batchvarov VN. Measurement, interpretation and clinical potential of QT dispersion. J Am Coll Cardiol. 2000;36:1749-1766. doi: 10.1016/s0735-1097(00)00962-1.

4. Kanbay M, Solak Y, Dogan E, et al. Uric Acid in Hypertension and Renal Disease: The Chicken or the Egg. Blood Purif. 2010;30:288-295. doi: 10.1159/000321074.

5. Ahmadnezhad M, Arefhosseini SR, Parizadeh MR, et al. Association between serum uric acid, high sensitive C-reactive protein and pro-oxidant-antioxidant balance in patients with metabolic syndrome. Biofactors. 2018;44:263-271. doi: 10.1002/biof.1424.

6. Grayson PC, Kim SY, LaValley M, et al. Hyperuricemia and incident hypertension: A systematic review and metaanalysis. Arthritis Care Res (Hoboken). 2011;63:102-110. doi: 10.1002/acr.20344.

7. Kim SY, Guevara JP, Kim KM, et al. Hyperuricemia and risk of stroke: A systematic review and meta-analysis. Arthritis and Rheumatism. 2009;61:885-892. doi: 10.1002/art.24612.

8. Kim SY, Guevara JP, Kim KM, et al. Hyperuricemia and coronary heart disease: a systematic review and metaanalysis. Arthritis Care Res (Hoboken). 2010;62:170-180. doi: 10.1002/acr.20065.

9. Kodama S, Saito K, Yachi Y, et al. Association between serum uric acid and development of type 2 diabetes. Diabetes Care. 2009;32:1737-1742. doi: 10.2337/dc09-0288.

10. Zhang JX, Lin X, Xu J, et al. Hyperuricemia Inhibition Protects SD Rats Against Fructose-Induced Obesity Hypertension Via Modulation of Inflammation and Renin-Angiotensin System in Adipose Tissue. Exp Clin Endocrinol Diabetes. 2021;129:314321. doi: 10.1055/a-1023-6710.

11. Krishnan E, Sokolove J. Uric acid in heart disease: a new C-reactive protein? Curr Opin Rheumatol. 2011;23:174-177. doi: 10.1097/BOR.ob013e3283432dd3.

12. Straus SM, Kors JA, De Bruin ML, et al. Prolonged QTc interval and risk of sudden cardiac death in a population of older adults. J Am Coll Cardiol. 2006;47:362-367. doi: 10.1016/j. jacc.2005.08.067.

13. Ryerson LM, Giuffre RM. QT intervals in metabolic dilated cardiomyopathy. Can J Cardiol. 2006;22:217-220. doi: 10.1016/ s0828-282x(06)70899-6.

14. Elming H, Holm E, Jun L, et al. The prognostic value of the QT interval and QT interval dispersion in all-cause and cardiac mortality and morbidity in a population of Danish citizens. Eur Heart J. 1998;19:1391-1400. doi: 10.1053/euhj.1998.1094.

15. Cuddy TE, Halli PS, Tate RB. QT dispersion and heart rate predict the risk of sudden unexpected cardiac death in men: the Manitoba Follow-Up Study. Prev Cardiol. 2009;12:27-33. doi: 10.1111/j.1751-7141.2008.00016.x.

16. Bazoukis G, Yeung C, Wui Hang Ho R, et al. Association of QT dispersion with mortality and arrhythmic events-A metaanalysis of observational studies. J Arrhythm. 2019;36:105115. doi: 10.1002/joa3.12253.

17. Brooksby P, Robinson PJ, Segal R, et al. Effects of losartan and captopril on QT dispersion in elderly patients with heart failure. ELITE study group. Lancet. 1999;534:395-396. doi: 10.1016/s0140-6736(99)01354-9.

18. Uchiyama K, Hayashi K, Fujino $\mathrm{N}$, et al. Impact of QT variables on clinical outcome of genotyped hypertrophic cardiomyopathy. Ann Noninvasive Electrocardiol. 2009;14:6571. doi: 10.1111/j.1542-474X.2008.00275.x.

19. Gertler MM, Garn SM, Levine SA. Serum uric acid in relation to age and physique in health and in coronary heart disease. Ann Intern Med. 1951;34:1421-1431. doi: 10.7326/0003-481934-6-1421.

20. Fang J, Alderman MH. Serum uric acid and cardiovascular mortality the NHANES I epidemiologic follow-up study, 19711992. National Health and Nutrition Examination Survey. JAMA. 2000;283:2404-2410. doi: 10.1001/jama.283.18.2404.

21. Puddu PE, Lanti M, Menotti A, et al. Gubbio Study Research Group. Serum uric acid for short-term prediction of cardiovascular disease incidence in the Gubbio population study. Acta Cardiol. 2001;56:243-251. doi: 10.2143/ AC.56.4.2005651.

22. Franse LV, Pahor M, Di Bari M, et al. Serum uric acid, diuretic treatment and risk of cardiovascular events in the Systolic Hypertension in the Elderly Program (SHEP). J Hypertens. 2000;18:1149-1154. doi: 10.1097/00004872-20001808000021.

23. Egas-Izquierdo $M$, Wong-Achi X, Alvarado-Villa G, et al. Relation between serum uric acid levels with the degree of coronary artery disease: A prospective study from Ecuador. Clin Investig Arterioscler. 2019;31:8-14. doi: 10.1016/j. arteri.2018.09.001.

24. Kanbay M, Ikizek M, Solak Y, et al. Uric acid and pentraxin-3 levels are independently associated with coronary artery disease risk in patients with stage 2 and 3 kidney disease. Am J Nephrol. 2011;33:325-331. doi: 10.1159/000324916.

25. Moriarity JT, Folsom AR, Iribarren C, et al. Serum uric acid and risk of coronary heart disease: Atherosclerosis Risk in Communities (ARIC) Study. Ann Epidemiol. 2000;10:136-143. doi: 10.1016/s1047-2797(99)00037-x.

26. Culleton BF, Larson MG, Kannel WB, et al. Serum uric acid and risk for cardiovascular disease and death: the Framingham Heart Study. Ann Intern Med. 1999;131:7-13. doi: 10.7326/0003-4819-131-1-199907060-00003.

27. Wannamethee SG. Serum uric acid is not an independent risk factor for coronary heart disease. Curr Hypertens Rep. 2001;3:190-196. doi: 10.1007/s11906-001-0037-3.

28. Sakata K, Hashimoto T, Ueshima H, et al. Absence of an association between serum uric acid and mortality from cardiovascular disease: NIPPON DATA 80, 1980-1994. Eur J Epidemiol. 2001;17:461-468. doi: 10.1023/a:1013735717961.

29. Naghavi M, John R, Naguib S, et al. pH Heterogeneity of human and rabbit atherosclerotic plaques; a new insight into detection of vulnerable plaque. Atherosclerosis. 2002;164:2735. doi: 10.1016/s0021-9150(02)00018-7. 
30. Hare JM, Johnson RJ. Uric acid predicts clinical outcomes in heart failure: insights regarding the role of xanthine oxidase and uric acid in disease pathophysiology. Circulation. 2003;107:1951-1953. doi: 10.1161/01.CIR.0000066420.36123.35.

31. Cappola TP, Kass DA, Nelson GS, et al. Allopurinol improves myocardial efficiency in patients with idiopathic dilated cardiomyopathy. Circulation. 2001;104:2407-2411. doi: 10.1161/hc4501.098928.

32. Farquharson CAJ, Butler R, Hill A, et al. Allopurinol improves endothelial dysfunction in chronic heart failure. Circulation. 2002;106:221-226. doi: 10.1161/01.cir.0000022140.61460.1d.

33. Doehner W, Schoene N, Rauchhaus $M$, et al. Effects of xanthine oxidase inhibition with allopurinol on endothelial function and peripheral blood flow in hyperuricemic patients with chronic heart failure: results from 2 placebo-controlled studies. Circulation. 2002;105:2619-2624. doi: 10.1161/01. cir.0000017502.58595.ed.

34. Khosla UM, Zharikov S, Finch JL, et al. Hyperuricemia induces endothelial dysfunction. Kidney Int. 2005;67:1739-1742. doi: 10.1111/j.1523-1755.2005.00273.x.

35. Mazzali M, Kanellis J, Han L, et al. Hyperuricemia induces a primary renal arteriolopathy in rats by a blood pressureindependent mechanism. Am J Physiol Renal Physiol. 2002;282:F991-997. doi: 10.1152/ajprenal.00283.2001.

36. Mazalli M, Hughes J, Kim YG, et al. Elevated uric acid increases blood pressure in the rat by a novel crystal-independent mechanism. Hypertension. 2001;38:1101-1106. doi: 10.1161/ hy1101.092839.

37. Waring WS, Adwani SH, Breukels O, et al. Hyperuricaemia does not impair cardiovascular function in healthy adults. Heart. 2004;90:155-159. doi: 10.1136/hrt.2003.016121.

38. Chamorro A, Planas AM. Yin and yang of uric acid in patients with stroke. Stroke. 2004;35:e11-e12. doi: 10.1161/01. STR.0000107762.79571.7D.

39. Cicero AF, Rosticci M, Tocci G, et al. Serum uric acid and other short-term predictors of electrocardiographic alterations in the Brisighella Heart Study cohort. Eur J Intern Med. 2015;26:255-258. doi: 10.1016/j.ejim.2015.02.007.

40. Özyılmaz S, Satılmışoğlu MH, Gül M, et al. Evaluation of the association between serum uric acid level andthe predicted risk score of sudden cardiac death in five years in patients with hypertrophic cardiomyopathy. Turk Kardiyol Dern Ars. 2018;46:111-120. doi: 10.5543/tkda.2017.60094.

41. Nodera M, Suzuki H, Matsumoto Y, et al. Association between SerumUric Acid Level andVentricularTachyarrhythmia in Heart Failure Patients with Implantable Cardioverter-Defibrillator. Cardiology. 2018;140:47-51. doi: 10.1159/000488851.

42. Yamada S, Suzuki H, Kamioka M, et al. Uric acid increases the incidence of ventricular arrhythmia in patients with left ventricular hypertrophy. Fukushima J Med Sci. 2012;58:101106. doi: 10.5387/fms.58.101. 